\title{
Extended Imaging Algorithm Based on Aperture Synthesis With Double-Scattered Waves for UWB Radars
}

\author{
Shouhei Kidera, Associate Member, IEEE, Takuya Sakamoto, Member, IEEE, and Toru Sato, Member, IEEE
}

\begin{abstract}
Ultrawideband (UWB) pulse radar with high range resolution is suitable for near-field sensing. Applications of UWB pulse radar include human body identification in blurry vision for security or rescue purposes and accurate spatial measurements for industrial products such as a reflector antenna. The synthetic aperture radar is still promising for these applications because it creates an accurate image even for near-field targets in free space. However, for complex-shaped or multiple objects, this algorithm suffers from increased shadow region because it employs only a single-scattered signal for imaging. To resolve this difficulty, this paper proposes a novel imaging algorithm based on aperture synthesis for double-scattered signals. In general, double-scattered waves include independent information on target points, which are not obtained by a single-scattered wave. Based on this principle, the proposed method effectively synthesizes the double-scattered signals and enhances the reconstructible range of a target shape, part of which becomes a shadow in the former approach. In order to enhance accuracy, a false image suppression approach based on the Fresnel zone theory is also incorporated in the proposed method. The results from numerical simulations and an experiment verify that our method significantly enhances the visible range of target surfaces without either a priori knowledge of target shapes or preliminary observation of their surroundings.
\end{abstract}

Index Terms-Complex-shaped or multiple targets, doublescattered waves, shadow region imaging, synthetic aperture radar (SAR), ultrawideband (UWB) radars.

\section{INTRODUCTION}

$\mathbf{N}$ ONCONTACT target reconstruction and localization with high spatial resolution can be accomplished by employing the ultrawideband (UWB) radar system. This type of radar overcomes an inherent problem in optical sensing that makes optical sensing hardly applicable to low-visibility situations or harsh-light conditions, such as dark smog, dusty environment, high-density gas, or strong backlight. As a typical application of the UWB radar, spatial measurements for security systems

Manuscript received August 25, 2009; revised April 30, 2010, October 13, 2010, December 22, 2010, and March 24, 2011; accepted May 20, 2011. Date of publication July 13, 2011; date of current version November 23, 2011. This work was supported in part by the Grant-in-Aid for Scientific Research (A) under Grant 17206044 and in part by the Grant-in-Aid for JSPS Fellows under Grant 19-497.

S. Kidera is with the Graduate School of Informatics and Engineering, The University of Electro-Communications, Chōfu 182-8585, Japan (e-mail: kidera@ee.uec.ac.jp).

T. Sakamoto and T. Sato are with the Department of Communications and Computer Engineering, Graduate School of Informatics, Kyoto University, Kyoto 606-8501, Japan (e-mail: t-sakamo@i.kyoto-u.ac.jp; tsato@kuee.kyotou.ac.jp).

Digital Object Identifier 10.1109/TGRS.2011.2158108 or rescue robots have potential for human body identification in darkness or in a disaster area, where a robot installed with array antennas on its body moves around the indoor environment. It is also applicable to the noncontact spatial measurement of industrial products with high precision and to specular surfaces such as reflector antennas or aircraft bodies. Moreover, it is promising for intruder detection or elderly care in a private room, where an optical camera generates the unavoidable problem of invasion of privacy.

Various kinds of radar algorithms have been developed that are aimed at geosurface measurement, land-mine detection, nondestructive testing, or indoor sensing. The classical studies based on aperture synthesis or range migration algorithms have been extensively applied to the aforementioned applications [1]-[5]. Recently, time-reversal methods have been developed for target detection in cluttered situations [6], [7] and for superresolution imaging by incorporating the multiple signal classification algorithm [8]. Some studies based on numerical solutions for domain integral equations have been developed by using multidimensional parametric optimization [9]-[11]. Different approaches aiming at clear boundary extraction are also promising for real-time and superresolution radar imaging by using the reversible boundary scattering transform between the range wavefront and the target boundary [12]. This is done either by calculating the envelope of spheres determined by the observed ranges [13], [14] or by directly reconstructing a complex-shaped target boundary using range point migration (RPM) [15]. However, they all have an inherent problem: The baseline length theoretically limits the reconstructible range of radar imagery. In many cases, the greater part of the target shape, such as the target side, falls into a shadow region that is never reconstructed because only single-scattered components are used for imaging.

As a substantial solution to this problem, the positive use of multiple-scattered signals has great potential for enhancing the imaging range. In general, the propagation path of a multiplescattered wave differs from that of a single-scattered one, which is determined by a stationary phase condition. This means that a multiple-scattered signal contains nontrivial information on target surfaces and thus has the potential to expand the visible range of radar imagery.

Several studies based on the analysis of multiple-scattered components have been reported. Bamler and Eineder [16] employed the double-bounced scattering effect to compensate for the synthetic aperture radar (SAR) images of the Gizeh 


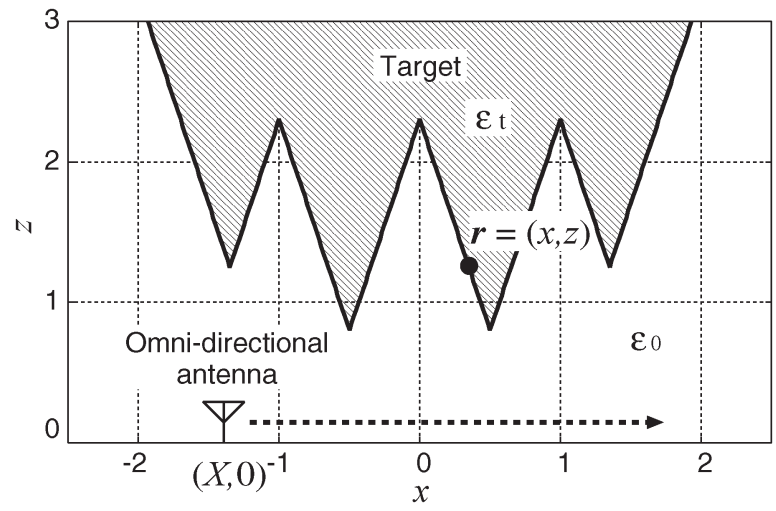

Fig. 1. System model in the 2-D problem.

pyramid, a part of which becomes a shadow. While it uses the positive phase of double-scattering waves, it does not build these components into the imaging algorithm.

Some algorithms based on a time-reversal approach using multiple-scattered signals have been proposed, which focus on reliable target detection or accurate positioning in cluttered situations [17]-[19]. While Moura and Jin [17] improved target detectability, it did not provide us with the target location or shape and the required a priori information about the surroundings or preliminary calibration. Shi and Nehorai [18] proved that the time-reversal solution including multiple-scattered signals could enhance the spatial resolution for multiple-point imaging. However, it specifies only the point target positioning and is not applicable to an arbitrary target boundary.

For a substantial solution to such difficulties, this paper proposes a shadow region imaging algorithm based on the aperture synthesis of the double-scattered signals. The SAR algorithm is still promising because it creates an accurate image even for near-field targets [1]. We extend the SAR algorithm so that it synthesizes the double-scattered signals by calculating the propagation path, in which the conventional SAR image is effectively utilized. This paper also introduces a postprocessing algorithm for false image reduction using the Fresnel zone, which is determined by another imaging algorithm such as RPM [15].

A novel outcome of our method is that it is applicable to arbitrary target shapes and makes a shadow region visible by using only single-observation data, in which preliminary calibration is not required. The proposed algorithm applicable to both the 2-D and 3-D problems is described in Section II. The results obtained from numerical simulation verify the effectiveness of the proposed method, which investigates examples with different target shapes, conductivity, or permittivity. Finally, an experimental study with the UWB modules proves that our method successfully expands the visible range of radar imagery.

\section{2-D PROBLEM}

\section{A. System Model}

Fig. 1 shows the system model in the 2-D problem. It assumes a monostatic radar system with an omnidirectional antenna that is scanned along the $x$-axis. It is assumed that the target has an arbitrary shape with a clear boundary. The propagation speed of the radio wave $c$ is assumed to be a known constant. A monocycle pulse is used as the transmitting current. The real space in which the target and antenna are located is expressed by the parameter $\boldsymbol{r}=(x, z)$ that is normalized by the central wavelength of the pulse $\lambda . z>0$ is assumed for simplicity. $s^{\prime}(X, t)$ is defined as the electric field received at the antenna location $(x, z)=(X, 0)$ at time $t . s(X, t)$ is calculated by applying the Wiener filter to $s^{\prime}(X, t)$ as

$$
s(X, t)=\int_{-\infty}^{\infty} W(\omega) S^{\prime}(X, \omega) e^{j \omega t} d \omega
$$

where $S^{\prime}(X, \omega)$ is the signal $s^{\prime}(X, t)$ in the frequency domain. $W(\omega)$ is defined as

$$
W(\omega)=\frac{S_{\mathrm{ref}}(\omega)^{*}}{(1-\eta) S_{0}^{2}+\eta\left|S_{\mathrm{ref}}(\omega)\right|^{2}} S_{0}
$$

where $\eta=1 /\left(1+(S / N)^{-1}\right)$ and $S_{\text {ref }}(\omega)$ is the reference signal in the frequency domain, which is the complex conjugate of the transmitted signal. $S_{0}$ is the constant for dimension consistency. The Wiener filter is an optimal mean-square-error linear filter for additive noises. $s(X, t)$ is converted into $s(X, Z)$ using the conversion $Z=c_{0} t / 2 \lambda$, where $c_{0}$ is the speed of the radio wave. This procedure is explained in more detail in [15].

\section{B. Conventional Algorithms}

1) SAR: SAR is a standard tool in radar imagery for applications such as geosurface measurement or soil-embedded landmine detection [1]. For spatial measurement in the near field, this algorithm is able to create an accurate image by using UWB signals. In the standard form of SAR, the distribution image $I_{1}(\boldsymbol{r})$ for the near field is formulated as

$$
I_{1}(\boldsymbol{r})=\int_{\boldsymbol{L} \in \Gamma} s\left(\boldsymbol{L}, d_{1}(\boldsymbol{r}, \boldsymbol{L}) / 2\right) d \boldsymbol{L}
$$

where $\boldsymbol{L}=(X, 0), \Gamma$ denotes the spatial range of the antenna scanning, and $d_{1}(\boldsymbol{r}, \boldsymbol{L})$ is the round-trip distance between $\boldsymbol{r}$ and $(X, 0)$. Equation (3) assumes a cylindrical incident wave and synthesizes the amplitudes of the received signals along each single-scattered path from the target boundary to the antenna location. The target boundary can be extracted from its focused image $I_{1}(\boldsymbol{r})$. This algorithm has the advantage of creating a significant image for arbitrary target shapes even in a noisy situation by using the complete information of the received signals $s(X, Z)[15]$.

Examples of this method are shown in the following using complex-shaped or multiple targets. Fig. 2 shows the outputs of the Wiener filter from the complex-shaped target shown in Fig. 1, where each signal is received at 101 locations for $-2.5 \leq X \leq 2.5$. The finite-difference time-domain method is used to calculate the received signal $s^{\prime}(X, Z)$. The conductivity and relative permittivity of the target are set to $1.0 \times 10^{6} \mathrm{~S} / \mathrm{m}$ and $\epsilon_{t}=1.0$, respectively, where the permittivity of air is $\epsilon_{0}=1.0$. These figures show that single-scattered waves are observed as positive peaks in the near range, whereas the parts 


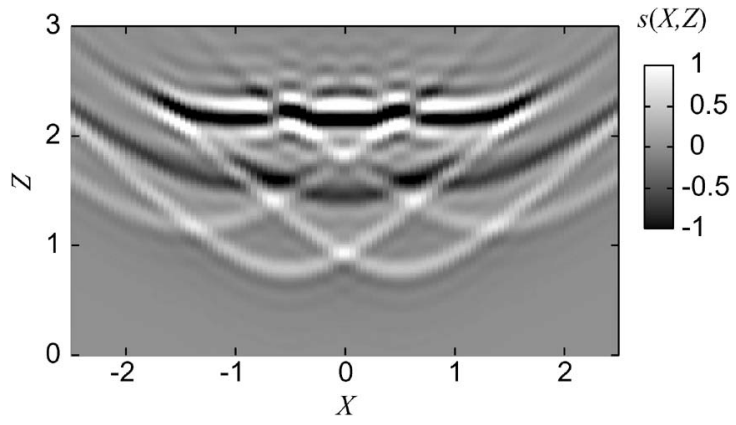

Fig. 2. Output of the Wiener filter $s(X, Z)$ from the complex-shaped target.

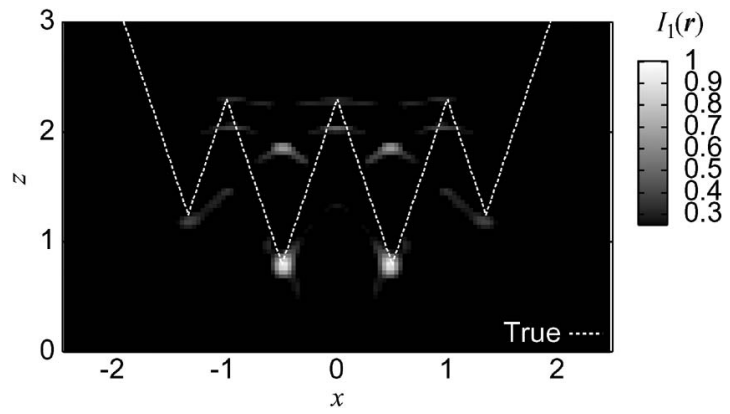

Fig. 3. Estimated image with the conventional SAR method $I_{1}(\boldsymbol{r})$ for the complex-shaped target.

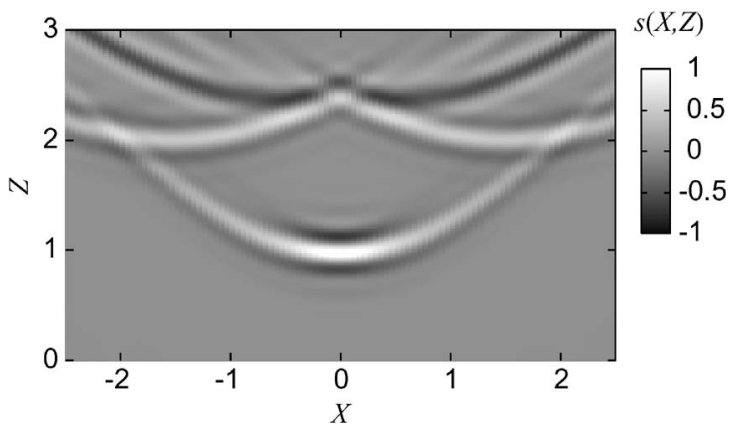

Fig. 4. Output of the Wiener filter $s(X, Z)$ from the multiple targets.

of the double-scattered waves are observed as negative peaks in the far range. Fig. 3 shows the images obtained by using the conventional SAR. The image is normalized by the maximum value of $I_{1}(\boldsymbol{r})$. This figure shows that the obtained image $I_{1}(\boldsymbol{r})$ expresses only sharp edges on the triangular boundaries and hardly reconstructs the other part of the target surface. Another case is presented in Fig. 4, which shows $s(X, Z)$ from multiple targets with circles and rectangular shapes. Fig. 5 shows the image with the same algorithm and illustrates a difficulty in imaging the side of the rectangular target because each antenna does not receive a direct echo from the side of each target boundary. This is an inherent problem in all algorithms that use only a single-scattered wave for target reconstruction.

2) Scattering Center Points of Single or Double Wave: This section investigates a simple analysis of the locations of scattered points by a geometrical optics approximation via single- or double-scattering waves. The spatial range of the reproduced target boundary is, in general, determined by the region of antenna scanning and the target shapes and locations. This is because an incident wave is intensively reflected in

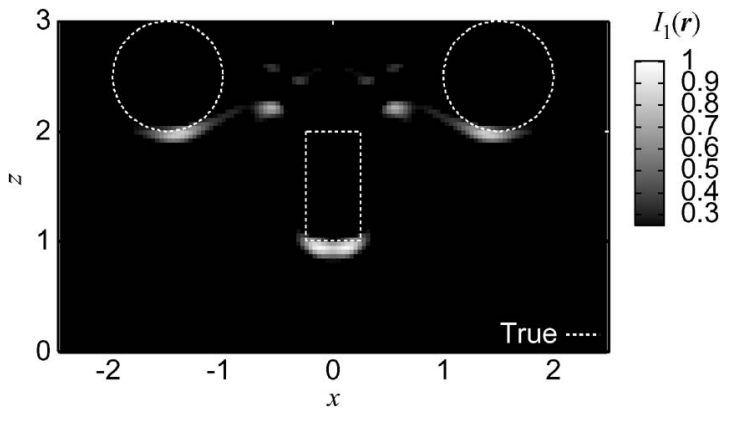

Fig. 5. Estimated image with the conventional SAR method $I_{1}(\boldsymbol{r})$ for the multiple targets.

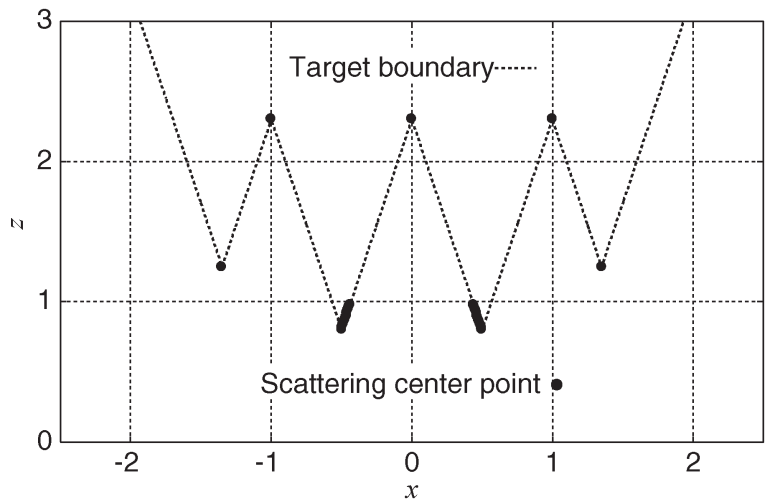

Fig. 6. Scattering center points of the single-scattered wave for the complexshaped target.

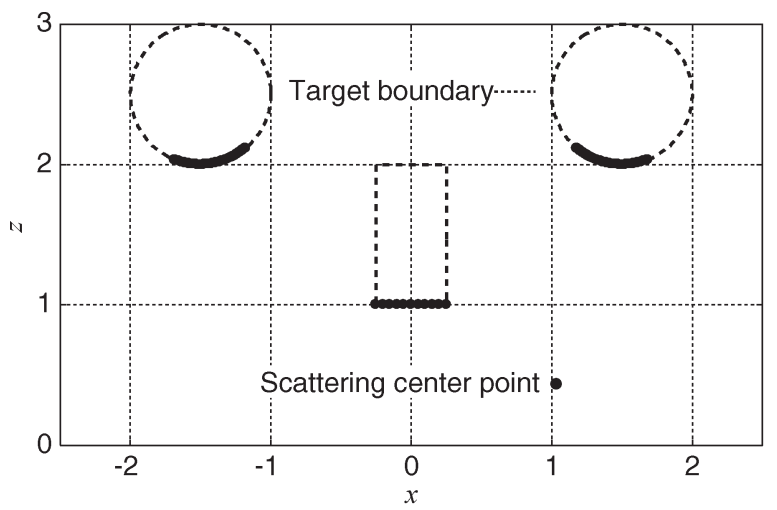

Fig. 7. Scattering center points of the single-scattered wave for the multiple targets.

the direction where a stationary phase condition is satisfied. For a single-scattered wave and a monostatic observation, an intensive echo is received from the direction perpendicular to the target boundary.

Figs. 6 and 7 show the scattering center points of a single-scattered wave for complex-shaped and multiple target boundaries, respectively. These points are calculated using geometrical optics considering that the propagation path is secluded from other targets. This calculation is explained in detail in Appendix A. Fig. 6 shows that the scattering center points express only the convex edges on the target boundary. Moreover, Fig. 7 shows that it is not possible to reproduce the side of the rectangular boundary. 


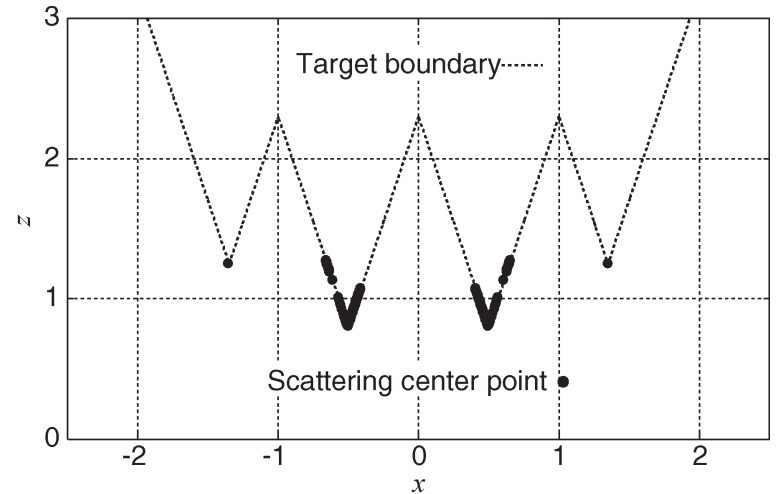

Fig. 8. Scattering center points of the double-scattered wave for the complexshaped target.

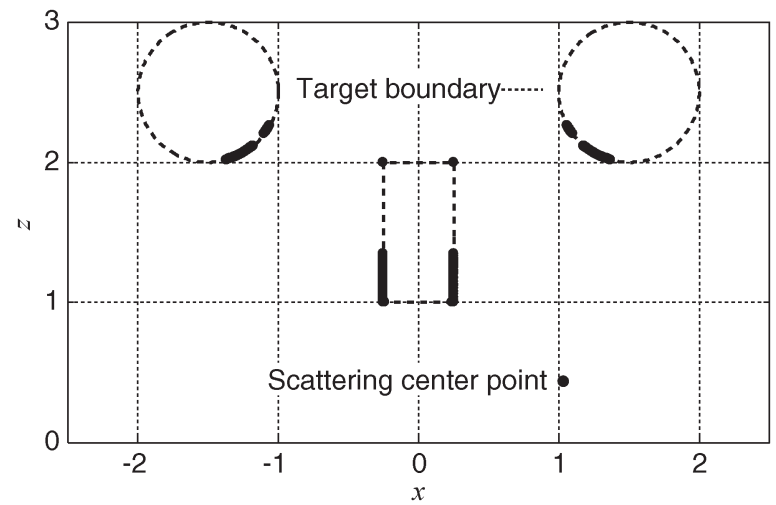

Fig. 9. Scattering center points of the double-scattered wave for the multiple targets.

In this paper, we define a shadow region as all the parts of the target boundaries that are not expressed as scattered center points in Figs. 6 and 7. This region always becomes invisible because only a single-scattered wave is used for imaging and the observation range is fixed. Also, the locations of the reproduced points in Fig. 3 do not strictly correspond to those in the focused image in Fig. 6 because the scattered wave has a frequency dependence and an amplitude variation that are not considered in the high-frequency approximation.

The scattering center points of the double-scattered waves are located by a similar approach, which is explained in detail in Appendix B. Fig. 8 shows the points reproduced by the doublescattered waves for the complex-shaped target. This figure confirms that the number of scattering center points substantially increases around the side of the triangular boundaries, the greater part of which is considered as a shadow region in Fig. 6. The reason is that many paths between the edge and the side of the triangle boundary satisfy the stationary phase condition of the double-scattered waves. Furthermore, Fig. 9 shows the scattering center points of the double-scattered waves for the multiple targets. It is confirmed that these points are located around the side of the rectangular target, thus making the region visible. Consequently, the positive use of a multiple-scattered component is promising for expanding the visible range of radar imagery.

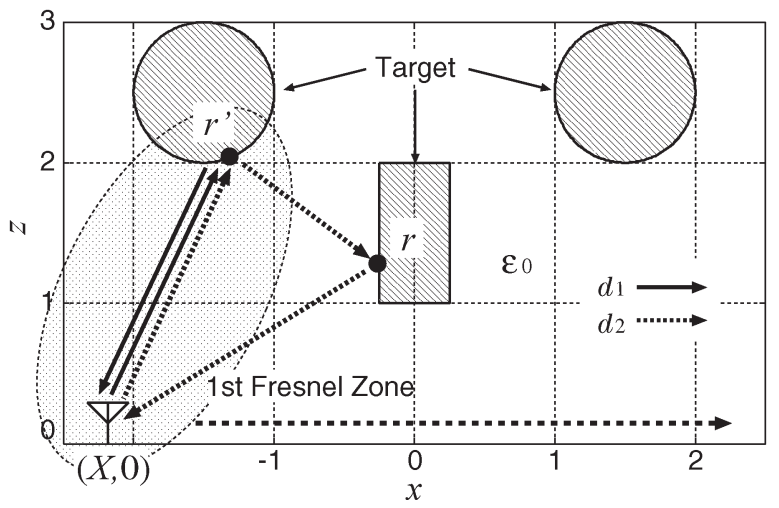

Fig. 10. Propagation paths of the single- and double-scattered waves and the first Fresnel zone.

\section{Proposed Algorithm}

This section proposes a shadow region imaging algorithm based on the aperture synthesis of the double-scattered waves. As previously mentioned, a double-scattered wave propagates with a path different from that of a single-scattered one. Therefore, it often provides invaluable information regarding the two target points, which are never obtained by a singlescattered wave. To enhance the visible range, the proposed method calculates the image $I_{2}(\boldsymbol{r})$ using the double-scattered waves as

$$
\begin{gathered}
I_{2}(\boldsymbol{r})=-\int_{\boldsymbol{r}^{\prime} \in R} \int_{\boldsymbol{L} \in \Gamma} I_{1}\left(\boldsymbol{r}^{\prime}\right) s\left(\boldsymbol{L}, d_{2}\left(\boldsymbol{r}, \boldsymbol{r}^{\prime}, \boldsymbol{L}\right) / 2\right) \\
\cdot F\left(\boldsymbol{r}, \boldsymbol{r}^{\prime}, \boldsymbol{L}\right) d \boldsymbol{L} d \boldsymbol{r}^{\prime}
\end{gathered}
$$

where $\boldsymbol{r}^{\prime}=\left(x^{\prime}, z^{\prime}\right), R$ denotes the region of real space, and $d_{2}\left(\boldsymbol{r}, \boldsymbol{r}^{\prime}, \boldsymbol{L}\right)=\sqrt{(x-X)^{2}+z^{2}}+\sqrt{\left(x^{\prime}-X\right)^{2}+z^{\prime 2}}+$ $\sqrt{\left(x-x^{\prime}\right)^{2}+\left(z-z^{\prime}\right)^{2}}$. The minus sign in (4) creates a positive image focused by the double-scattered waves that have an antiphase relationship with the single-scattered waves. $F\left(\boldsymbol{r}, \boldsymbol{r}^{\prime}, \boldsymbol{L}\right)$ is the weighting function defined as

$$
F\left(\boldsymbol{r}, \boldsymbol{r}^{\prime}, \boldsymbol{L}\right)=1-\exp \left[-\frac{\left\{d_{2}\left(\boldsymbol{r}, \boldsymbol{r}^{\prime}, \boldsymbol{L}\right)-d_{1}\left(\boldsymbol{r}^{\prime}, \boldsymbol{L}\right)\right\}^{2}}{2 \sigma_{\mathrm{FZ}}^{2}}\right]
$$

where $\sigma_{\mathrm{FZ}}=0.5 \lambda$ is set, and $F\left(\boldsymbol{r}, \boldsymbol{r}^{\prime}, \boldsymbol{L}\right)$ suppresses the images inside the first Fresnel zone. This is because $I_{2}(\boldsymbol{r})$ often includes unnecessary images caused by the range sidelobe of $s(X, Z)$, particularly in front of the actual target boundaries. Fig. 10 shows an example of $\boldsymbol{r}$ and $\boldsymbol{r}^{\prime}$ on the double-scattering paths and the first Fresnel zone. The weighting function in (4) physically means that the region included in the first Fresnel zone is regarded as an unreasonable candidate for the existing target region. This is because the first Fresnel zone is defined as the spatial area that dominantly contributes the electromagnetic wave propagation from the transmitting to the receiving antennas. This algorithm regards the first scattering point $\boldsymbol{r}^{\prime}$ focused by the initial image $I_{1}\left(\boldsymbol{r}^{\prime}\right)$ as the reradiation source, and the first Fresnel zone determined by this source $r^{\prime}$ and the antenna location $(X, 0)$, as shown in Fig. 10, should not include another target point of the double scattering. 
If $\sigma_{\mathrm{FZ}}$ is much smaller than $0.5 \lambda, I(\boldsymbol{r})$ probably contains the image caused by the range sidelobe of the received signal. In contrast, if $\sigma_{\mathrm{FZ}}$ is much greater than $0.5 \lambda$, the intensity of the focused image by the double-scattered waves around $r$, as shown in Fig. 10, becomes smaller because the spatial size of the suppression becomes considerably large.

Equation (4) expresses the aperture synthesis of the received signals by considering double-scattered paths. This algorithm assumes that one of the double-scattering center points is included in the initial image $I_{1}(\boldsymbol{r})$. This assumption is mostly approved in the case of general target shapes, particularly with edges that often become the scattering point of both the single and double reflections. If either point of the doublescattering centers is focused in $I_{1}(\boldsymbol{r})$, the region around the other scattering point is reproduced in $I_{2}(\boldsymbol{r})$ in (4).

In this paper, we assume that the positive images of $I_{1}(\boldsymbol{r})$ and $I_{2}(\boldsymbol{r})$ are necessary for target boundary extraction. Then, the proposed method determines the final image $I(\boldsymbol{r})$ as

$$
I^{\prime}(\boldsymbol{r})=\frac{I_{1}(\boldsymbol{r}) H\left(I_{1}(\boldsymbol{r})\right)}{\max _{\boldsymbol{r}} I_{1}(\boldsymbol{r})}+\frac{I_{2}(\boldsymbol{r}) H\left(I_{2}(\boldsymbol{r})\right)}{\max _{\boldsymbol{r}} I_{2}(\boldsymbol{r})}
$$

where $H(x)$ is the Heaviside function with

$$
H(x)= \begin{cases}1, & (x \geq 0) \\ 0, & (x<0)\end{cases}
$$

Equation (6) creates the image that is focused by both the images $I_{1}(\boldsymbol{r})$ and $I_{2}(\boldsymbol{r})$, providing a more significant target image.

In addition, we introduce a false image reduction algorithm to enhance the accuracy of the obtained image $I^{\prime}(\boldsymbol{r})$. It uses an image obtained by the RPM method, which accurately extracts the target boundary points from the observed range points [15]. First, this method extracts the group of range points defined as $\left(X_{i}, Z_{i}\right)\left(i=1, \ldots, N_{R}\right)$ from the local peaks of $s(X, Z)$, where $N_{R}$ denotes the total number of range points. Next, the RPM transforms the group of range points defined as $\left(X_{i}, Z_{i}\right)$ into the group of target points defined as $\boldsymbol{r}_{i}^{\mathrm{rpm}}$ using an accurate direct-of-arrival estimation. The penalty function for each $\boldsymbol{r}_{i}^{\mathrm{rpm}}$ is defined as

$$
\begin{aligned}
\zeta\left(\boldsymbol{r}_{i}^{\mathrm{rpm}}\right)= & \frac{1}{N_{R}} \sum_{j=1}^{N_{R}} \exp \left\{-\frac{d_{1}\left(\boldsymbol{r}_{j}^{\mathrm{rpm}}, \boldsymbol{L}_{i}\right)^{2}}{2 \sigma\left(Z_{i}\right)^{2}}\right\} \\
& \times \exp \left\{-\frac{\left(d_{2}\left(\boldsymbol{r}_{i}^{\mathrm{rpm}}, \boldsymbol{r}_{j}^{\mathrm{rpm}}, \boldsymbol{L}_{i}\right)-d_{1}\left(\boldsymbol{r}_{i}^{\mathrm{rpm}}, \boldsymbol{L}_{i}\right)\right)^{2}}{2 \sigma_{\mathrm{FZ}}^{2}}\right\}
\end{aligned}
$$

where $\sigma\left(Z_{i}\right)=Z_{i} / 2$ and $\boldsymbol{L}_{i}=\left(X_{i}, 0\right) . \zeta\left(\boldsymbol{r}_{i}^{\mathrm{rpm}}\right)$ increases when the first Fresnel zone determined by $\boldsymbol{r}_{i}^{\mathrm{rpm}}$ and $\boldsymbol{L}_{i}$ includes other target points. Fig. 11 shows the target points obtained by the RPM method and the example of the first Fresnel zone for the multiple targets. In this figure, the area around which $\zeta\left(\boldsymbol{r}_{i}^{\mathrm{rpm}}\right)$ increases should be considered as inappropriate for imaging because there are many other target points in its first

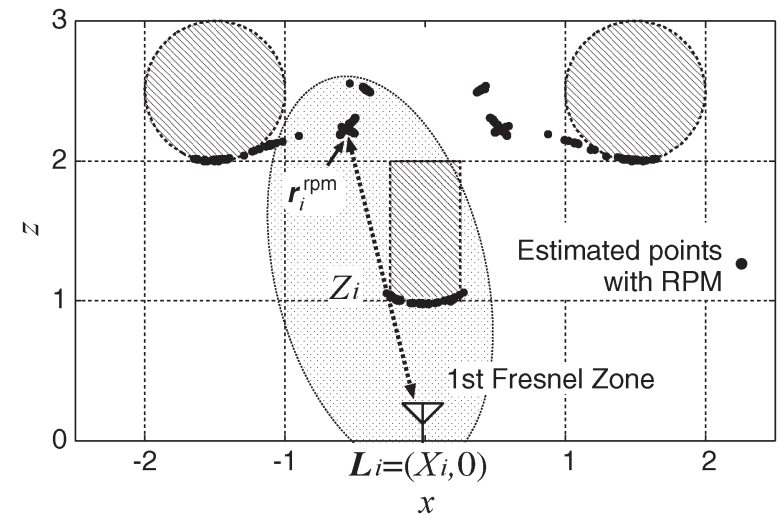

Fig. 11. Target points obtained by the RPM algorithm and the first Fresnel zone.

Fresnel zone. Such a situation is unrealistic for actual radar imaging [12]. Next, the filtering function $G(\boldsymbol{r})$ is defined as

$$
G(\boldsymbol{r})=G_{0} \sum_{j=1}^{N_{R}} \zeta\left(\boldsymbol{r}_{i}^{\mathrm{rpm}}\right) \exp \left\{-\frac{\left|\boldsymbol{r}_{i}^{\mathrm{rpm}}-\boldsymbol{r}\right|^{2}}{2 \sigma_{r}^{2}}\right\} .
$$

Then, the final image $I(\boldsymbol{r})$ is determined as

$$
I(\boldsymbol{r})= \begin{cases}I^{\prime}(\boldsymbol{r}) / G(\boldsymbol{r}), & (G(\boldsymbol{r})>1) \\ I^{\prime}(\boldsymbol{r}), & (G(\boldsymbol{r}) \leq 1)\end{cases}
$$

where the constants $\sigma_{r}$ and $G_{0}$ are empirically determined.

Finally, the summary of the actual procedure of the proposed method is given as follows.

Step 1) The initial image as $I_{1}(\boldsymbol{r})$ is calculated in (3) using the output of the Wiener filter $s(X, Z)$.

Step 2) Equation (4) creates image $I_{2}(\boldsymbol{r})$ using the initial image $I_{1}(\boldsymbol{r})$, and (6) synthesizes them as $I^{\prime}(\boldsymbol{r})$.

Step 3) The penalty function $\zeta\left(\boldsymbol{r}_{i}^{\mathrm{rpm}}\right)$ is calculated by (8) for each target point $\boldsymbol{r}_{i}^{\mathrm{rpm}}$, which is preliminarily estimated by the RPM method.

Step 4) Equations (9) and (10) determine the final image $I(\boldsymbol{r})$.

The proposed method uses only the initial image $I_{1}(\boldsymbol{r})$ and emphasizes the target regions scattered by the doublescattered waves. This means that it does not require a priori knowledge of the target shape or preliminary observation of the surrounding environment. This is a definitive advantage over other algorithms using multiple-scattered signals [16]-[19].

\section{Performance Evaluation in Numerical Simulation}

This section refers to the examples of the proposed method for both the target cases introduced in Section II-B.1. Fig. 12 shows the image $I_{2}(\boldsymbol{r})$ for the complex-shaped target by using the same data as in Fig. 2. $I_{2}(\boldsymbol{r})$ is normalized by its maximum value. This figure shows that a portion of the target side can be reconstructed with the double-scattered waves. Fig. 13 shows $G(\boldsymbol{r})$ calculated in (9), which is determined by the penalty function $\zeta\left(\boldsymbol{r}_{i}^{\mathrm{rpm}}\right)$ in (8) using the preliminarily estimated points with RPM. In this case, $\sigma_{r}=0.2 \lambda$ is set. As shown in this figure, $G(\boldsymbol{r})$ increases around the backside of the actual boundary for $-1.0 \leq x \leq 1.0$ and $1.8 \leq z \leq 2.0$, where the false images can 


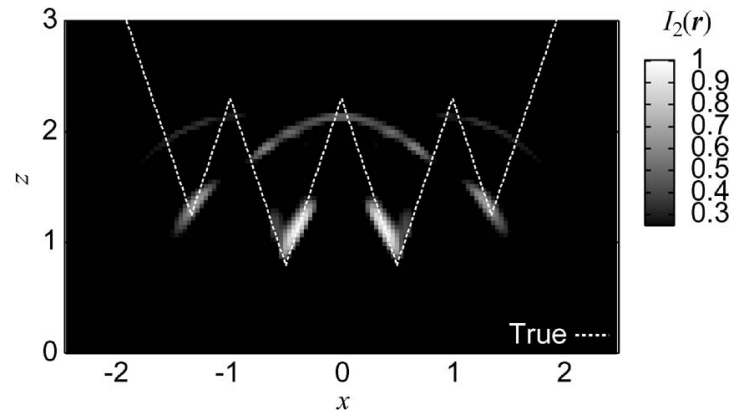

Fig. 12. Estimated image with the double-scattered waves $I_{2}(\boldsymbol{r})$ for the complex-shaped target.

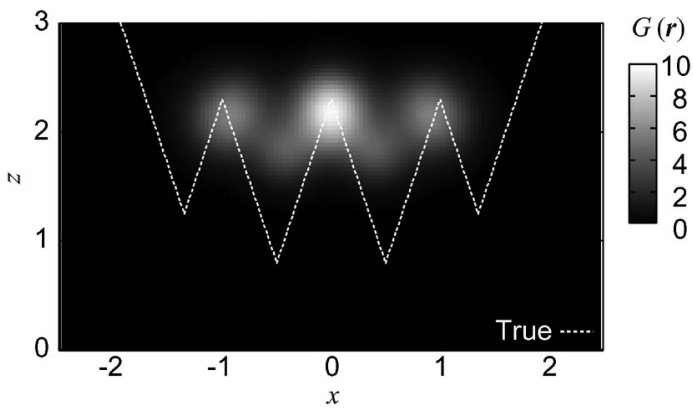

Fig. 13. False image reduction filter $G(\boldsymbol{r})$ for the complex-shaped target.

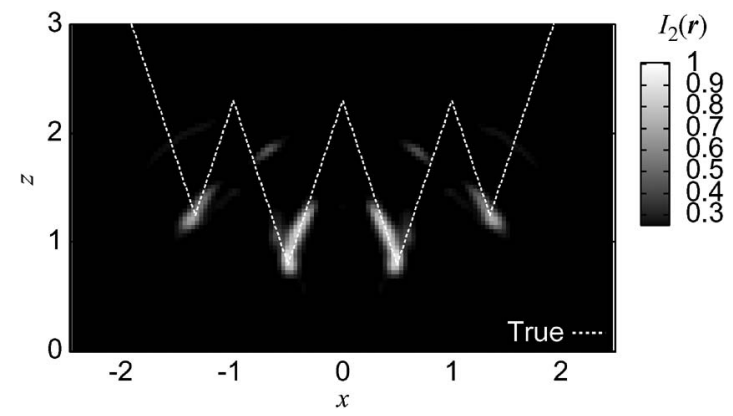

Fig. 14. Estimated image with the double-scattered waves $I(\boldsymbol{r})$ for the complex-shaped target.

be recognized, as in Figs. 3 and 12. This is because the RPM creates several target points with higher $\zeta\left(\boldsymbol{r}_{i}^{\mathrm{rpm}}\right)$ corresponding to multiple-scattered centers. Fig. 14 shows the final image after applying false image reduction filter with $G(\boldsymbol{r})$ in (10), where $G_{0}=1$ is set. This provides us with an essential and accurate image to recognize multiple triangular structures. Fig. 15 shows the estimated image $I_{2}(\boldsymbol{r})$ for multiple objects using the same data as in Fig. 4. Although it can reconstruct the rectangular side of the target, false images are found between the circular and rectangular boundaries. Fig. 16 shows $G(\boldsymbol{r})$ determined by the target points with RPM, as shown in Fig. 11. $G(\boldsymbol{r})$ also increases around $0.4 \leq|x| \leq 0.6$ and $2.2 \leq z \leq 2.5$, where the false images can also be seen in Figs. 5 and 15. Fig. 17 shows the final image $I(\boldsymbol{r})$ and reveals that the visible ranges of the circular and rectangular boundaries are significantly expanded by simultaneously suppressing the false images. As a further note, the false images seen around $1.6 \leq|x| \leq 2.0$ and $1.0 \leq$ $z \leq 1.8$ are comparatively reduced by synthesizing both $I_{1}(\boldsymbol{r})$ and $I_{2}(\boldsymbol{r})$ in (6). This is because the double-scattered waves

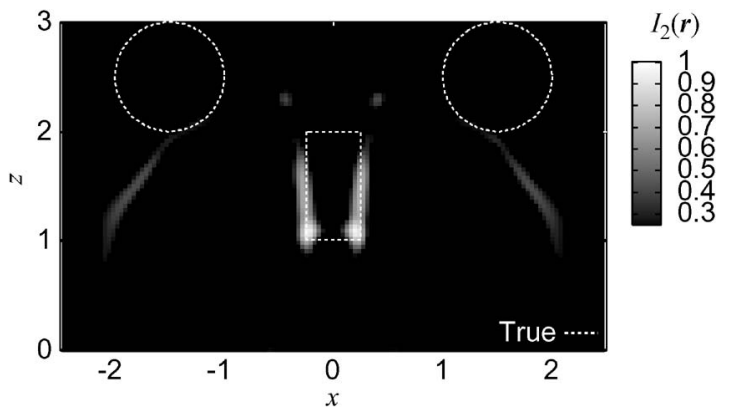

Fig. 15. Estimated image with the double-scattered waves $I_{2}(\boldsymbol{r})$ for the multiple targets.

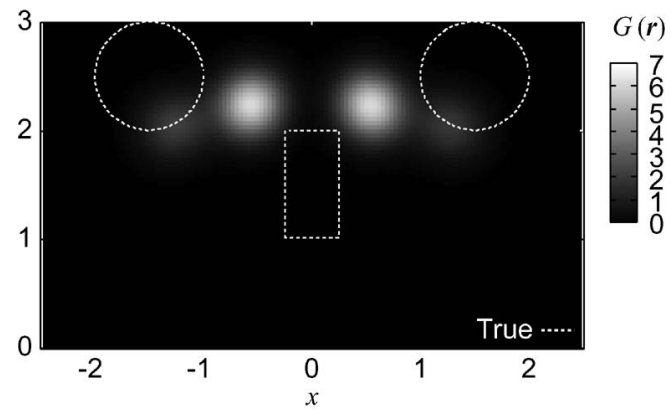

Fig. 16. False image reduction filter $G(\boldsymbol{r})$ for the multiple targets.

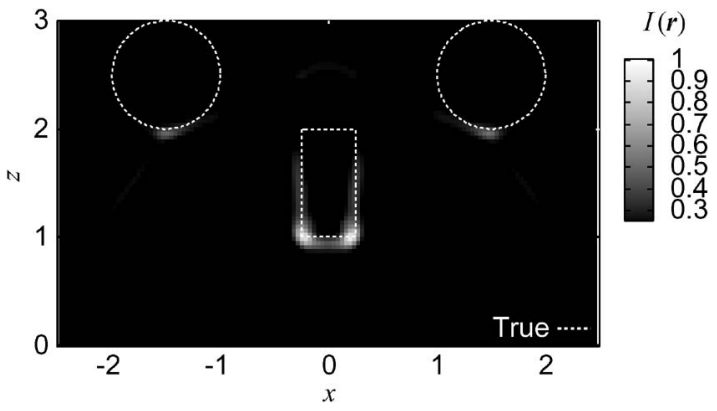

Fig. 17. Estimated image with the double-scattered waves $I(\boldsymbol{r})$ for the multiple targets.

are effectively focused on the part of the target side in (4). Moreover, it should be noted that this method does not require target modeling or a priori information of the surroundings, and yet, this is a noticeable advantage over conventional algorithms [17]-[19]. However, if both the regions scattered by the doublescattered waves are invisible in the initial image $I_{1}(\boldsymbol{r})$, those regions should fall into a shadow region even by using this method. The aforementioned problems are topics for future study.

For the quantitative analysis of the estimated images, an evaluation value for imaging accuracy is introduced as

$$
\bar{\epsilon}=\frac{\int_{\boldsymbol{r} \in R} \min _{\boldsymbol{r}_{\text {true }}}\left\|\boldsymbol{r}_{\text {true }}-\boldsymbol{r}\right\|\left|I^{\prime}(\boldsymbol{r})\right|^{2} d x d y}{\int_{\boldsymbol{r} \in R}\left|I^{\prime}(\boldsymbol{r})\right|^{2} d x d y}
$$

where $I^{\prime}(\boldsymbol{r})$ is the estimated image and $\boldsymbol{r}_{\text {true }}$ denotes the locations of the actual target boundaries. $\bar{\epsilon}$ increases when the image focuses the region far from the actual target boundary, 


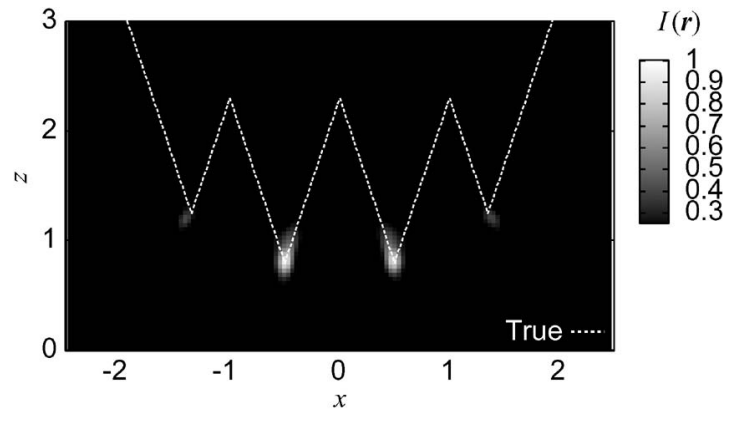

Fig. 18. Estimated image with the proposed method $I(\boldsymbol{r})$ for the complexshaped target in the noisy case at $S / N=20 \mathrm{~dB}$.

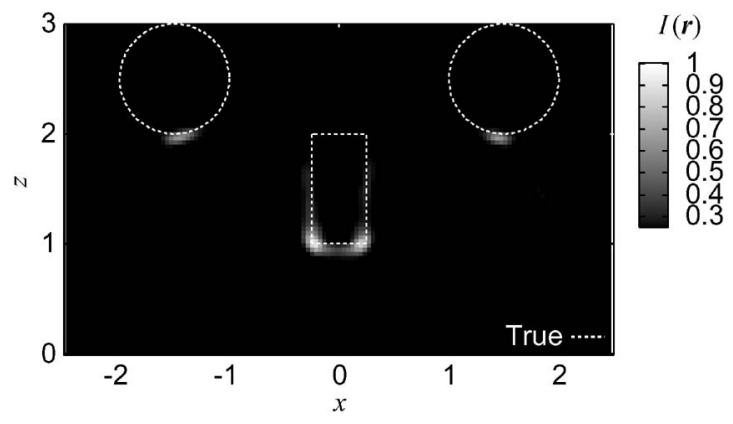

Fig. 19. Estimated image with the proposed method $I(\boldsymbol{r})$ for the multiple targets in the noisy case at $S / N=20 \mathrm{~dB}$.

and a small $\bar{\epsilon}$ verifies the accurate target boundary extraction. For the complex-shaped objects, as in Figs. 3 and 14, $\bar{\epsilon}$ 's for $I_{1}(\boldsymbol{r})$ and $I(\boldsymbol{r})$ are $0.163 \lambda$ and $0.113 \lambda$, respectively. For the multiple targets, as in Figs. 5 and 17, $\bar{\epsilon}$ 's of $I_{1}(\boldsymbol{r})$ and $I(\boldsymbol{r})$ are $0.217 \lambda$ and $0.205 \lambda$, respectively. This evaluation proves that the proposed method enhances the imaging range without significant accuracy deterioration.

Next, examples in the noisy situation are demonstrated. White noise is added to $s^{\prime}(X, Z)$. Figs. 18 and 19 show the estimated images with the proposed method for the complexshaped and multiple targets, respectively. The $S / N$ ratios of the double-scattered signals are $20 \mathrm{~dB}$ in both cases. The $S / N$ ratio is defined as the ratio of the peak instantaneous signal power to the average noise power after applying the matched filter with the transmitted waveform. While the magnitudes of the focused images are relatively weak compared to those in noiseless situations due to image dispersion by random noise, each boundary obtained still shows its shadow region. $\bar{\epsilon}$ 's for $I(\boldsymbol{r})$ as in Figs. 18 and 19 are $0.156 \lambda$ and $0.206 \lambda$, respectively.

To verify the applicability of the proposed method, examples for different target characteristics are presented as follows. Fig. 20 shows the estimated image obtained by the proposed method, where the conductivity and relative permittivity of the targets are set as $\sigma=2.0 \mathrm{~S} / \mathrm{m}$ and $\epsilon_{t}=50$, respectively. These parameters correspond to the average values of those with a human body around frequencies in the range $1-5 \mathrm{GHz}$ [20]. It shows that the estimated image $I(\boldsymbol{r})$ still shows the part of the target side. This is because the reflection signals caused by the transparent waves through the targets are received at a relatively far range, and its focused image appears in the region away from the actual targets. $\bar{\epsilon}$ for $I(\boldsymbol{r})$ in this case is $0.126 \lambda$. Fig. 21

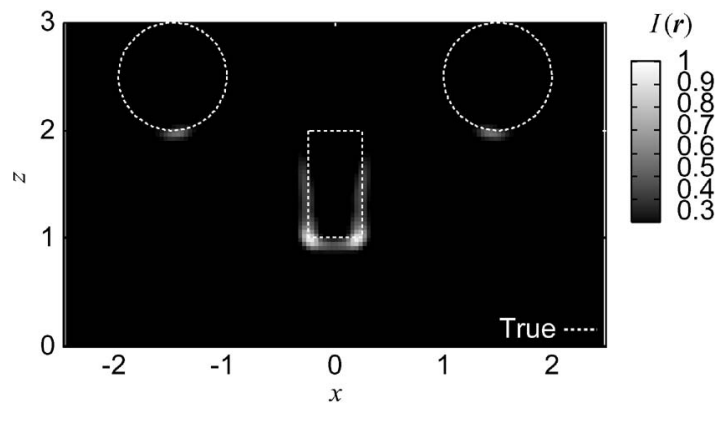

Fig. 20. Estimated image with the proposed method $I(\boldsymbol{r})$ for the multiple targets with $\sigma=2.0 \mathrm{~S} / \mathrm{m}$ and $\epsilon_{t}=50$.

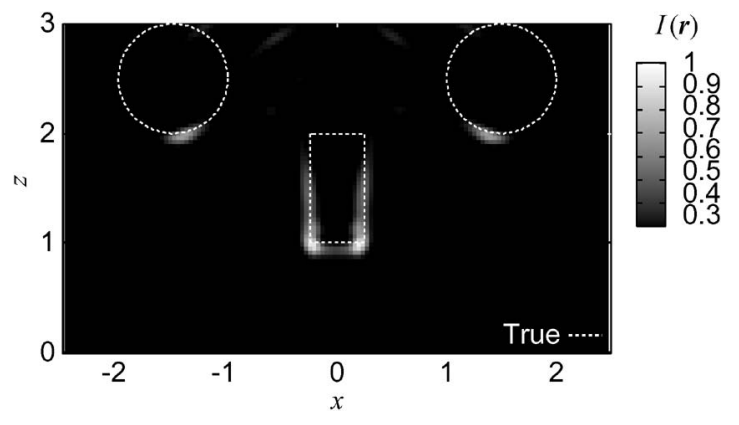

Fig. 21. Estimated image with the proposed method $I(\boldsymbol{r})$ for the multiple targets with $\sigma=0.005 \mathrm{~S} / \mathrm{m}$ and $\epsilon_{t}=5.0$.

also shows the estimated image obtained by the proposed method where the conductivity and relative permittivity are set as $\sigma=0.005 \mathrm{~S} / \mathrm{m}$ and $\epsilon_{t}=5.0$, respectively. These parameters are equivalent to those of concrete walls. It is confirmed that the part of the target side is still reconstructed. $\bar{\epsilon}$ for $I(\boldsymbol{r})$ in this case is $0.208 \lambda$.

Next, an example of the transmitted pulse with narrower bandwidth is investigated to establish the practicability of the proposed method. In general, the image quality obtained by the conventional and proposed SARs depends on the transmitted waveform. The fractional bandwidth (FBW) is the essential factor determining the spatial resolution of the obtained image because the real space is normalized by the center wavelength of the transmitted pulse as $\lambda$. According to the Federal Communications Commission, the FBW of the UWB signal is defined as

$$
F B W=\frac{2\left(f_{\mathrm{High}}-f_{\text {Low }}\right)}{f_{\text {High }}+f_{\text {Low }}}
$$

where $f_{\text {High }}$ is the highest frequency limit with a signal $10 \mathrm{~dB}$ below peak emission and $f_{\text {Low }}$ is the lowest frequency limit with a signal $10 \mathrm{~dB}$ below peak emission.

The FBW in the former simulation is around $150 \%$ because it uses a monocycle pulse for the transmitted signal. In order to compare with the experimental investigation described in Section III-C, where the FBW of the transmitted signal is estimated to be around $65 \%$, an example of the narrower bandwidth signal is presented as follows. Fig. 22 shows the estimated image where the FBW of the transmitted signal is set to $75 \%$. This figure shows that the estimated images deteriorate from that in Fig. 17 in the case of wider bandwidth. This is because 


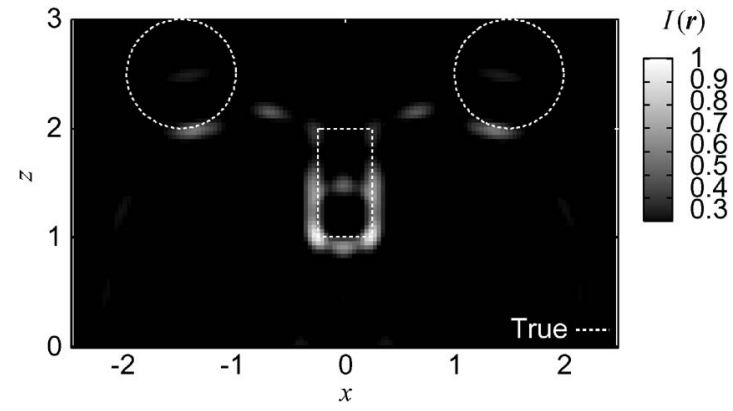

Fig. 22. Estimated image with the proposed method $I(\boldsymbol{r})$ for the multiple targets, where the FBW of the transmitted pulse is $75 \%$.

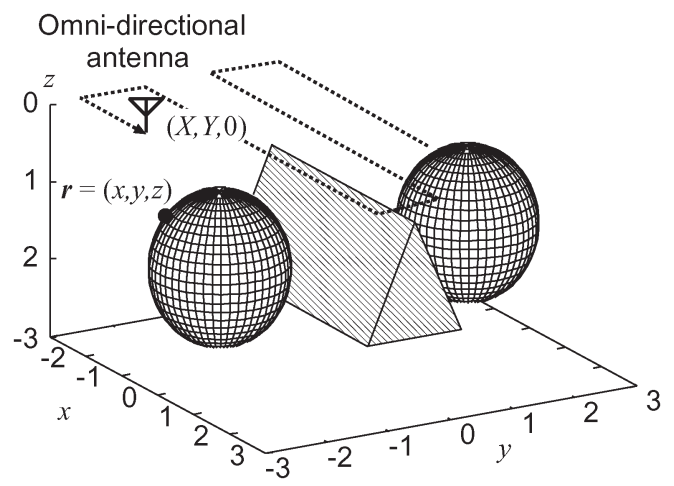

Fig. 23. System model in the 3-D problem.

the lower FBW causes a higher range sidelobe of the Wiener filter, which produces the images away from the actual target boundary. Then, the accuracy is relatively distorted, where $\bar{\epsilon}$ for $I(\boldsymbol{r})$ is $0.290 \lambda$. Consequently, the bandwidth of the transmitted signal is an essential factor determining the image quality of the proposed method.

Furthermore, this method requires triple integration of the received signals in (4), which requires around $30 \mathrm{~min}$ of calculation with a Xeon $2.8-\mathrm{GHz}$ processor. Thus, the acceleration of the imaging speed is also required as a future study.

\section{3-D PROBLEM}

\section{A. System Model and Imaging Algorithm}

Fig. 23 shows the system model for the 3-D problem. The target model, antenna, and transmitted signal are the same as those assumed in the 2-D problem. The antenna is scanned on the plane $z=0$. Linear polarization in the direction of the $x$ axis is assumed. The $r$-space is expressed by the parameter $r=$ $(x, y, z)$, and $z>0$ is assumed for simplicity. $s\left(X, Y, Z^{\prime}\right)$ is defined as the output of the Wiener filter at the antenna location $(X, Y, 0)$ in the $r$-space.

In the 3-D problem, the distribution image $I_{1}(\boldsymbol{r})$ with the conventional SAR is calculated, where the variables in (3) are redefined as $\boldsymbol{r}=(x, y, z)$ and $\boldsymbol{L}=(X, Y, 0)$ and $d_{1}(\boldsymbol{r}, \boldsymbol{L})$ is defined as the round-trip distance between the target point $r$ and the antenna location $(X, Y, 0)$. The proposed algorithm is also extended to the 3-D model by redefining the variables in (4)-(6), where $\boldsymbol{r}^{\prime}=\left(x^{\prime}, y^{\prime}, z^{\prime}\right)$, and $d_{2}\left(\boldsymbol{r}, \boldsymbol{r}^{\prime}, \boldsymbol{L}\right)$ is defined as the peripheral distance of the triangle whose apexes are

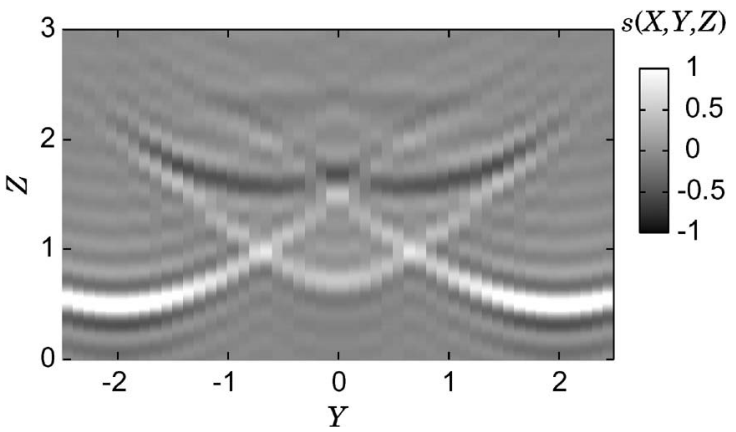

Fig. 24. Output of the Wiener filter $s(X, Y, Z)$ from the multiple targets at $X=0$.

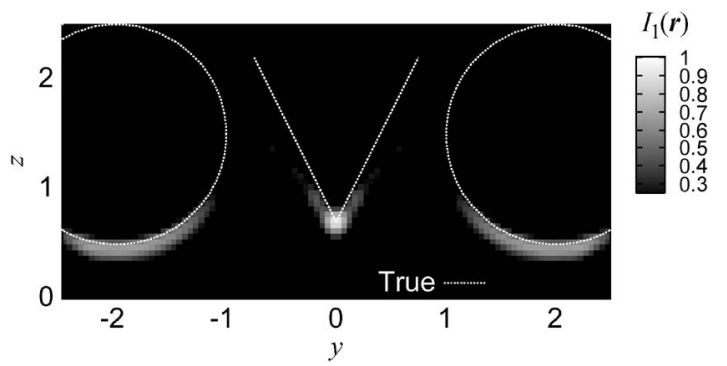

Fig. 25. Estimated image with the conventional SAR method $I_{1}(\boldsymbol{r})$ for the multiple targets at $x=0$.

$\boldsymbol{r}, \boldsymbol{r}^{\prime}$, and the antenna location $(X, Y, 0)$. The false image reduction algorithm is also incorporated in the proposed method by using the target points created by RPM in the 3-D model [15]. Although the proposed method in the 3-D model requires fifth-order integration and greater calculation compared to the 2-D model, it accomplishes nonparametric 3-D imaging for the shadow region without preliminary observation by synthesizing the double-scattered components.

\section{B. Performance Evaluation in Numerical Simulation}

This section investigates the performance evaluation of each method using numerical simulation. First, the examples of the conventional SAR are presented as follows. Fig. 24 shows the output of the Wiener filter in the cross section $X=0$. The multiple targets shown in Fig. 23 are assumed. The received signals are observed for $-2.5 \leq X$ and $Y \leq 2.5$ at 51 locations for each axis. Fig. 25 shows the cross section of the estimated image at $x=0$ obtained by the conventional SAR. This figure shows that the image expresses only the bottom part of the target boundary and never provides the triangular shapes. Another case is demonstrated when a complex-shaped boundary is assumed as shown in Fig. 26. Fig. 27 shows the output of the Wiener filter in the cross section $X=0$ in this case. Fig. 28 shows the reconstructed image of the complex-shaped target at $x=0$. Imaging around acute convex boundaries cannot be performed by this method. This is because the direct-scattered wave from the target side is not received at any antenna location for the same reason as mentioned in the 2-D problem.

Next, examples with the proposed method are shown. Fig. 29 shows the estimated image at the $x=0$ view with the proposed method for the multiple targets, as in Fig. 23. Fig. 30 shows the 


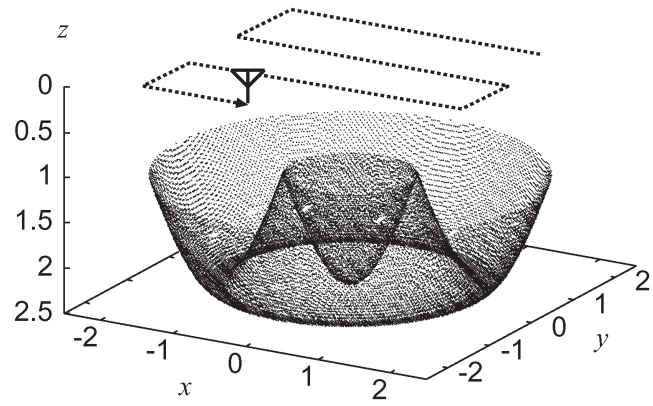

Fig. 26. True target boundary in the complex-shaped target.

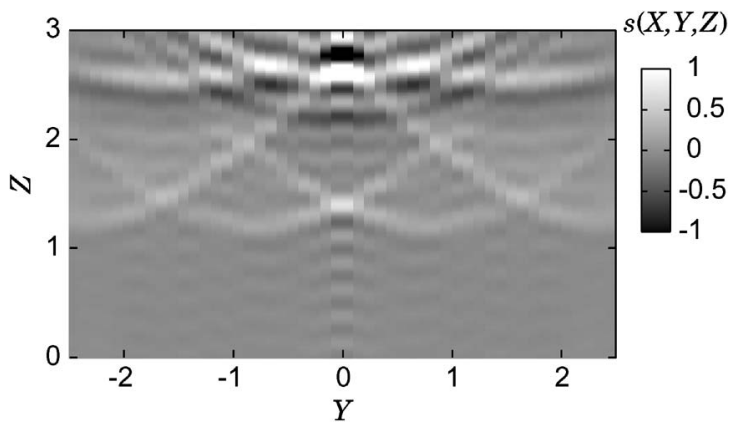

Fig. 27. Output of the Wiener filter $s(X, Y, Z)$ from the complex-shaped targets at $X=0$.

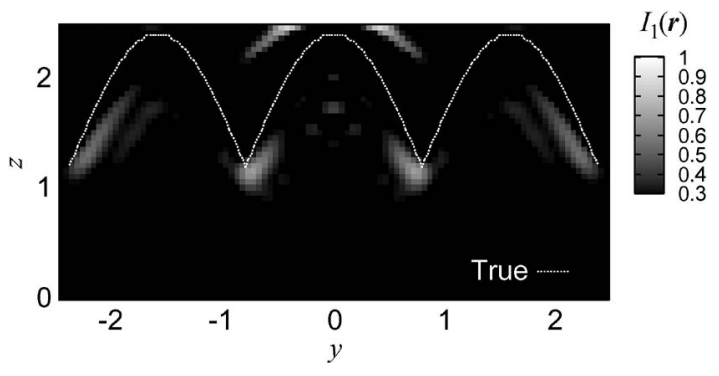

Fig. 28. Estimated image with the conventional SAR method $I_{1}(\boldsymbol{r})$ for the complex-shaped target at $x=0$.

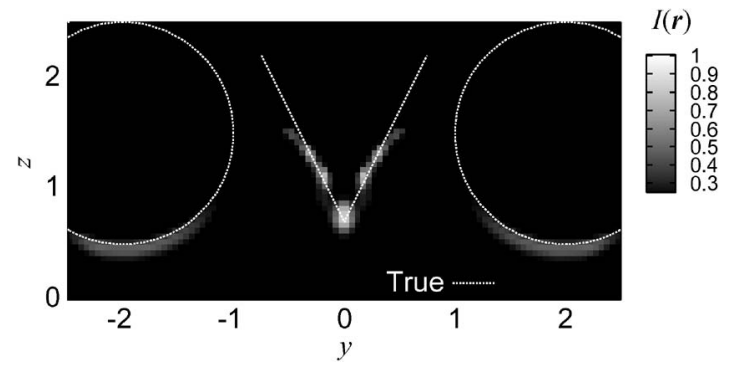

Fig. 29. Estimated image with the proposed method $I(\boldsymbol{r})$ for the multiple objects at $x=0$.

estimated image $I(\boldsymbol{r})$ at the cross section $z=1$.0. It reveals that the proposed method expands the spatial range of the visible region and makes the part of the triangular side visible even in the 3-D problem. $\bar{\epsilon}$ 's defined by (11) of the estimated images $I_{1}(\boldsymbol{r})$ and $I(\boldsymbol{r})$ are $0.175 \lambda$ and $0.082 \lambda$, respectively, in this case. Fig. 31 shows the view identical to that in Fig. 29 for the complex-shaped boundary, as shown in Fig. 26. Fig. 32 shows the estimated image $I(\boldsymbol{r})$ at the cross section $z=1.5$.

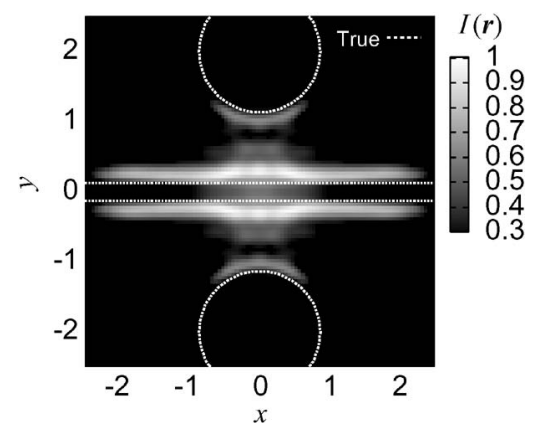

Fig. 30. Estimated image with the proposed method $I(\boldsymbol{r})$ for the multiple objects at $z=1.0$.

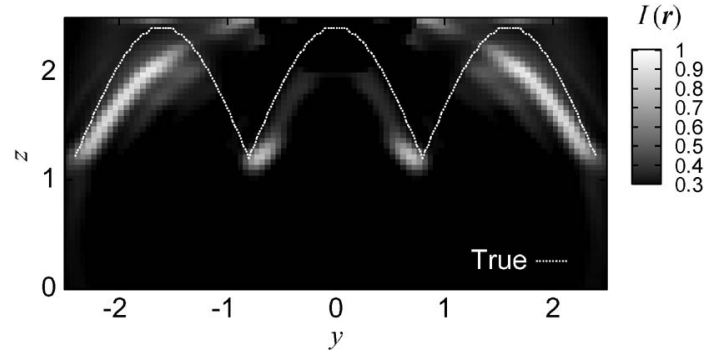

Fig. 31. Estimated image with the proposed method $I(\boldsymbol{r})$ for the complexshaped target at $x=0$.

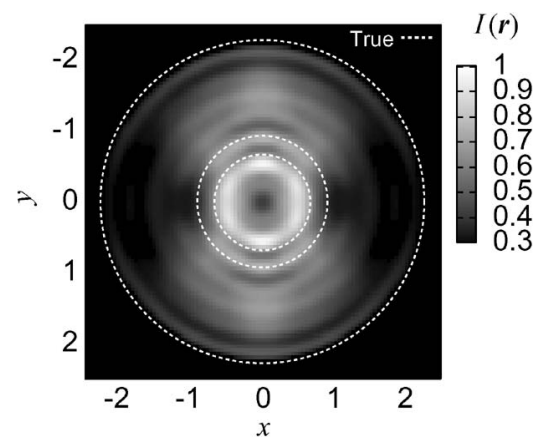

Fig. 32. Estimated image with the proposed method $I(\boldsymbol{r})$ for the complexshaped target at $z=1.5$.

This figure verifies the effectiveness of the proposed method in providing knowledge to identify the original shape. This is because the double-scattered waves are effectively focused on the rectangular side in (4). In addition, $\bar{\epsilon}$ 's for the estimated images $I_{1}(\boldsymbol{r})$ and $I(\boldsymbol{r})$ in this case are $0.185 \lambda$ and $0.242 \lambda$, respectively. These results also verify quantitatively that the proposed method retains its accuracy in expanding the reconstructible range in the $3-\mathrm{D}$ problem.

However, each cross section required around $100 \mathrm{~min}$ of calculation with a Xeon $2.8-\mathrm{GHz}$ processor. Reduction in processing time should be addressed in a future study.

\section{Performance Evaluation in Experiment}

This section presents the experimental investigation of the proposed algorithm. Fig. 33 shows the experimental setup including the geometrical arrangement for transmitting and receiving antennas and multiple targets. A UWB pulse with a center frequency of $3.3 \mathrm{GHz}$ and a $10-\mathrm{dB}$ bandwidth of $3.0 \mathrm{GHz}$ 


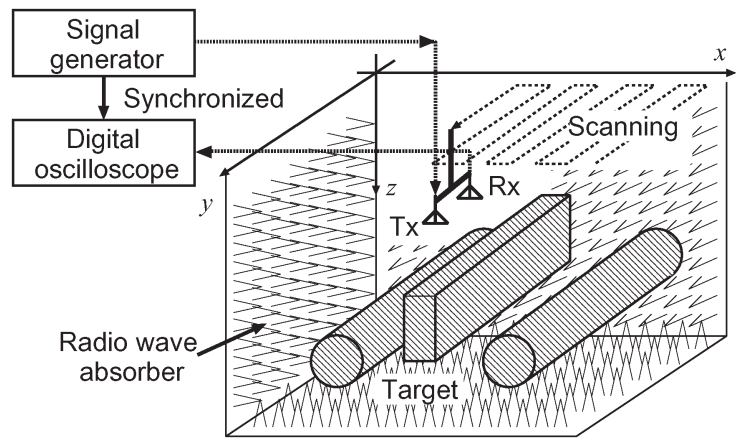

Fig. 33. Experimental setup.

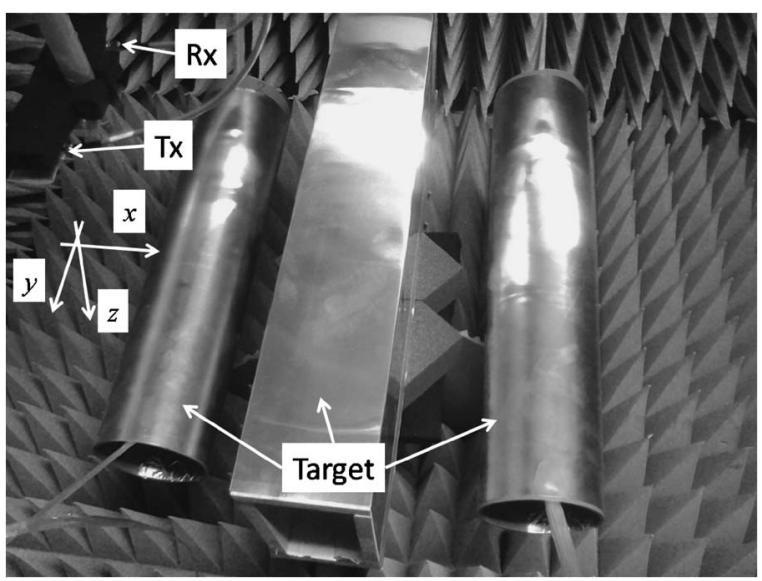

Fig. 34. Arrangement of the pair of antennas and multiple targets in the anechoic chamber.

is transmitted with the signal generator. The pair of transmitting and receiving microstrip patch antennas is scanned on the $z=0$ plane for $-300 \mathrm{~mm} \leq x \leq 300 \mathrm{~mm}$ and $-100 \mathrm{~mm} \leq$ $y \leq 100 \mathrm{~mm}$, respectively, with both sampling intervals set to $10 \mathrm{~mm}$. The separation between the transmitting and receiving antennas is $126 \mathrm{~mm}$ in the direction of the $y$-axis. The data in the time domain are coherently averaged 1024 times using a digital oscilloscope. Fig. 34 shows the actual arrangement of the antennas and the cylindrical and rectangular targets made of stainless steel sheets located in an anechoic chamber.

The direct-scattered signal $s^{\prime}\left(X, Y, Z^{\prime}\right)$ from the targets can be obtained by eliminating the received signal in the absence of the target. First, we measure the direct wave entering from the transmitting antenna at the location of $\left(X_{0}, Y_{0}, 0\right)$ in the absence of the target defined as $r_{\text {notgt }}\left(X_{0}, Y_{0}, Z^{\prime}\right)$ and eliminate this component from the received signals $r_{\mathrm{tgt}}\left(X, Y, Z^{\prime}\right)$ observed in the presence of the target. Then, the signals $s^{\prime}\left(X, Y, Z^{\prime}\right)$ including only the scattered components from the target are calculated as

$$
s^{\prime}\left(X, Y, Z^{\prime}\right)=r_{\mathrm{tgt}}\left(X, Y, Z^{\prime}\right)-r_{\text {notgt }}\left(X_{0}, Y_{0}, Z^{\prime}\right) .
$$

In this experiment, the direct wave from the transmitting antenna is regarded to have the same waveform for all antenna locations because the interval of the transmitting and receiving antennas is fixed.

Fig. 35 shows the output of the Wiener filter at $Y=0$ in the experiment. Fig. 36 shows the estimated image at $y=0$

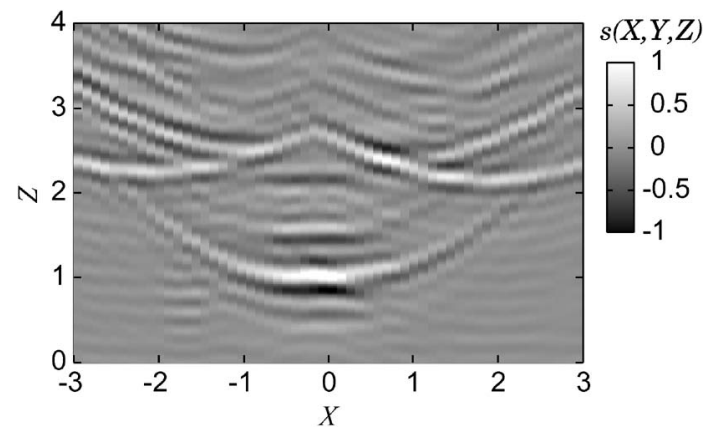

Fig. 35. Output of the Wiener filter in the experiment $s(X, Y, Z)$ at $Y=0$.

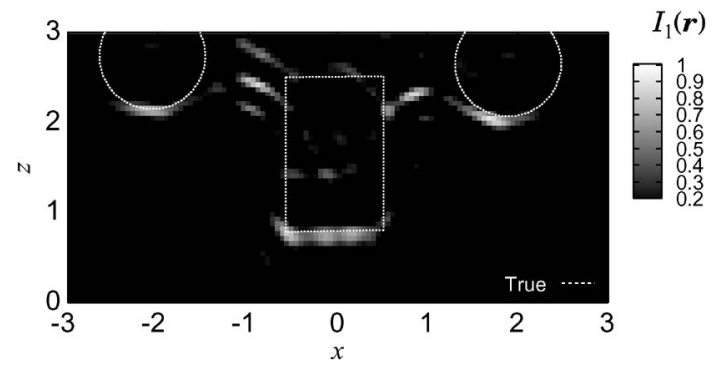

Fig. 36. Estimated image with the conventional SAR method $I_{1}(\boldsymbol{r})$ in the experiment at $y=0$.

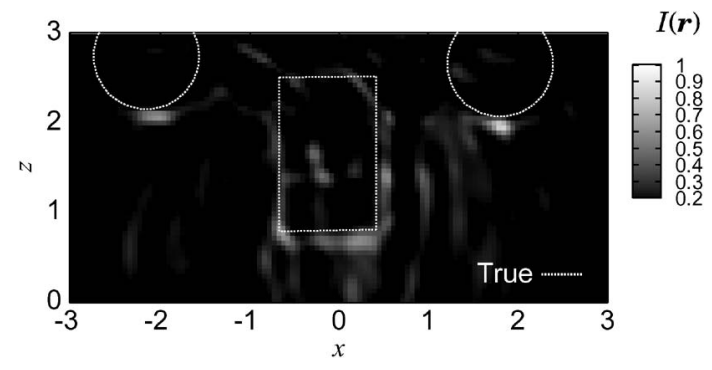

Fig. 37. Estimated image with the proposed method $I(\boldsymbol{r})$ in the experiment at $y=0$.

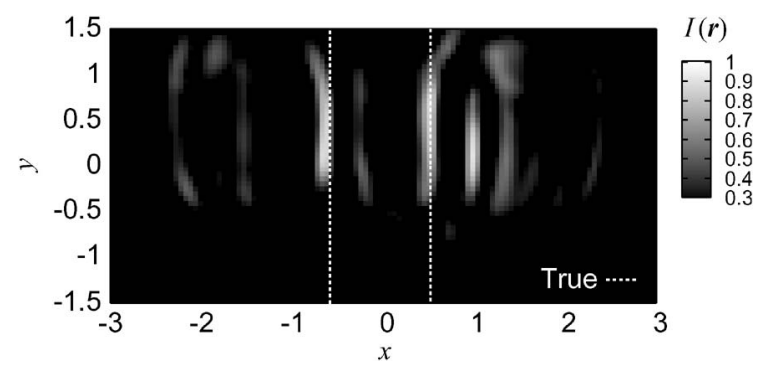

Fig. 38. Estimated image with the proposed method $I(\boldsymbol{r})$ in the experiment at $z=1.6$

with the conventional SAR method. This figure shows that only the bottom part of the target boundary is reconstructed, which corresponds well to the result of the numerical simulation in Fig. 5. On the contrary, Fig. 37 shows the estimated image with the proposed method at $y=0$. In this case, the bistatic extension of the proposed method is applied. It offers an expanded target image along the rectangular side and provides significant information about the rectangular target boundary even in a real environment. Fig. 38 also shows the estimated image with the 
proposed method at the cross section $z=1.6$. As defined in (11), $\bar{\epsilon}$ 's of the estimated images $I_{1}(\boldsymbol{r})$ and $I(\boldsymbol{r})$ in this case are $0.236 \lambda$ and $0.410 \lambda$, respectively. There are some false images around the side of the rectangular target that are focused by the range sidelobes of the signals because the transmitted signal has a narrower FBW, defined in (12), of approximately $65 \%$ compared with $150 \%$ assumed in the numerical simulation. The false image reduction algorithm described in Section II-C is not effective for these false images because the lower FBW substantially causes an image distortion as shown in Fig. 22 and discussed in Section II-D. This is an unavoidable characteristic of actual radar systems. However, it is not a substantial problem in the proposed method because a wider band signal and antenna can suppress these false images. The calculation time in this case is around $100 \mathrm{~min}$ for each cross-sectional image. It will be promising to introduce the frequency-wavenumber $(F-K)$ migration into the proposed method to enhance the processing speed [21], [22].

\section{CONCLUSiON}

This paper has proposed a shadow region imaging algorithm based on the aperture synthesis for double-scattered waves, which incorporates false image suppression based on the Fresnel zone theory. The conventional SAR suffers from increased shadow regions for complex-shaped or multiple targets because it only uses a single-scattered signal for imaging. The geometrical optics approximation proves that the doublescattered waves are useful for enhancing the visible range of target images. Numerical simulations and an experiment verified that the proposed method could obtain the greater part of the complex-shaped or multiple targets, which could not be obtained by the conventional SAR. Moreover, this method has a substantial advantage over other methods in that it does not require a priori information of target models or preliminary observation of the surroundings. We also confirmed that the image obtained by the proposed method created an accurate image in a noisy situation, where the $S / N$ ratios of the double-scattered signals were greater than $20 \mathrm{~dB}$. The additional examples in the numerical simulation also verify that the proposed algorithm is applicable to general dielectric objects, such as a human body or a concrete wall, in specifying target boundary extraction. Although the proposed method requires numerous calculations, it has the potential to expand the application range of near-field radar in a multiple-scattering environment.

It should be noted that this paper only provides the expansion of the imaging range by using double-scattered components. For the detection or recognition of a human body, a car, or an airplane, another study such as pattern recognition or data clustering with the preliminary data is necessary. In addition, for indoor or urban sensing, there are many objects that cause multiple scatterings, such as walls, buildings, or trees. According to the results in this paper, the positive use of these multiple-scattered waves has great potential for enhancing the visible range including the blind area of an optical camera or a conventional sensor system.

\section{APPENDIX}

\section{A. A. Derivation of Target Points on Single-Scattered Paths}

This section derives the path which the single-scattered wave propagates. The location of a visible point is defined as $\boldsymbol{p}_{\mathrm{s}}$. Based on the geometrical optics approximation, $\boldsymbol{p}_{\mathrm{s}}$ is calculated as

$$
\boldsymbol{p}_{\mathrm{s}}=\left\{(x, z) \mid(X, 0) \in \Gamma, \partial d_{1}(\boldsymbol{r}, X) / \partial x=0\right\} .
$$

In addition, the point $\boldsymbol{p}_{\mathrm{s}}$ satisfies the following condition that a propagation path is secluded by the target region $T_{i}$ as

$$
s \boldsymbol{p}_{\mathrm{s}}+(1-s) \boldsymbol{p}_{\mathrm{X}} \notin \bigcup_{i=1}^{N} T_{i}
$$

where $\boldsymbol{p}_{\mathrm{X}}=(X, 0)$, the inner region of the targets is defined as $(x, z) \in T_{i}$, and $N$ denotes the number of targets. $s$ is a variable for $0 \leq s \leq 1$.

\section{B. B. Derivation of Target Points on Double-Scattered Paths}

Next, the paths of the double-scattered waves are derived as follows. The pair of two scattering points on the target is defined as $\boldsymbol{p}_{\mathrm{s}}$ and $\boldsymbol{p}_{\mathrm{d}}$. The geometrical optics gives the condition which the pair points satisfy as

$$
\begin{aligned}
& \left(\boldsymbol{p}_{\mathrm{s}}, \boldsymbol{p}_{\mathrm{d}}\right) \\
& \quad=\left[\left(x, z, x^{\prime}, z^{\prime}\right) \mid(X, 0) \in \Gamma,\left\{\begin{array}{l}
\partial d_{2}\left(x, z, x^{\prime}, z^{\prime}, X\right) / \partial x=0 \\
\partial d_{2}\left(x, z, x^{\prime}, z^{\prime}, X\right) / \partial x^{\prime}=0
\end{array}\right] .\right.
\end{aligned}
$$

Moreover, $\boldsymbol{p}_{\mathrm{s}}$ and $\boldsymbol{p}_{\mathrm{d}}$ should satisfy the seclusion condition from the targets, formulated as

$$
\left.\begin{array}{l}
s \boldsymbol{p}_{\mathrm{s}}+(1-s) \boldsymbol{p}_{\mathrm{X}} \\
s \boldsymbol{p}_{\mathrm{X}}+(1-s) \boldsymbol{p}_{\mathrm{d}} \\
s \boldsymbol{p}_{\mathrm{d}}+(1-s) \boldsymbol{p}_{\mathrm{s}}
\end{array}\right\} \notin \bigcup_{i=1}^{N} T_{i} .
$$

\section{REFERENCES}

[1] D. L. Mensa, G. Heidbreder, and G. Wade, "Aperture synthesis by object rotation in coherent imaging," IEEE Trans. Nucl. Sci., vol. NS-27, no. 2, pp. 989-998, Apr. 1980.

[2] J. Song, Q. H. Liu, P. Torrione, and L. Collins, "Two-dimensional and three dimensional NUFFT migration method for landmine detection using ground-penetrating radar," IEEE Trans. Geosci. Remote Sens., vol. 44, no. 6, pp. 1462-1469, Jun. 2006.

[3] F. Soldovieri, A. Brancaccio, G. Prisco, G. Leone, and R. Pieri, "A Kirchhoff-based shape reconstruction algorithm for the multimonostatic configuration: The realistic case of buried pipes," IEEE Trans. Geosci. Remote Sens., vol. 46, no. 10, pp. 3031-3038, Oct. 2008.

[4] X. Zhuge, A. G. Yarovoy, T. Savelyev, and L. Ligthart, "Modified Kirchhoff migration for UWB MIMO array-based radar imaging," IEEE Trans. Geosci. Remote Sens., vol. 48, no. 6, pp. 2692-2703, Jun. 2008.

[5] A. Brancaccio and G. Leone, "Multimonostatic shape reconstruction of two-dimensional dielectric cylinders by a Kirchhoff-based approach," IEEE Trans. Geosci. Remote Sens., vol. 48, no. 8, pp. 3152-3161, Aug. 2008

[6] D. Liu, G. Kang, L. Li, Y. Chen, S. Vasudevan, W. Joines, Q. H. Liu, J. Krolik, and L. Carin, "Electromagnetic time-reversal imaging of a target in a cluttered environment," IEEE Trans. Antennas Propag., vol. 53, no. 9, pp. 3058-3066, Sep. 2005. 
[7] D. Liu, J. Krolik, and L. Carin, "Electromagnetic target detection in uncertain media: Time-reversal and minimum-variance algorithms," IEEE Trans. Geosci. Remote Sens., vol. 45, no. 4, pp. 934-944, Apr. 2007.

[8] E. A. Marengo, F. K. Gruber, and F. Simonetti, "Time-reversal MUSIC imaging of extended targets," IEEE Trans. Image Process., vol. 16, no. 8, pp. 1967-1984, Aug. 2007.

[9] A. Massa, D. Franceschini, G. Franceschini, M. Pastorino, M. Raffetto, and M. Donelli, "Parallel GA-based approach for microwave imaging applications," IEEE Trans. Antennas Propag., vol. 53, no. 10, pp. 31183127, Oct. 2005.

[10] M. Donelli, D. Franceschine, P. Rocca, and A. Massa, "Three-dimensional microwave imaging problems solved through an efficient multiscaling particle swarm optimization," IEEE Trans. Geosci. Remote Sens., vol. 47, no. 5, pp. 1467-1481, May 2009.

[11] G. P. Otto and W. C. Chew, "Microwave inverse scattering-Local shape function imaging for improved resolution of strong scatterers," IEEE Trans. Microw. Theory Tech., vol. 42, no. 1, pp. 137-141, Jan. 1994.

[12] T. Sakamoto and T. Sato, "A target shape estimation algorithm for pulse radar systems based on boundary scattering transform," IEICE Trans. Commun., vol. E87-B, no. 5, pp. 1357-1365, May 2004.

[13] S. Kidera, T. Sakamoto, and T. Sato, "High-resolution and real-time UWB radar imaging algorithm with direct waveform compensations," IEEE Trans. Geosci. Remote Sens., vol. 46, no. 11, pp. 3503-3513, Nov. 2008.

[14] T. C. Williams, J. M. Sill, and E. C. Fear, "Breast surface estimation for radar-based breast imaging system," IEEE Trans. Biomed. Eng., vol. 44, no. 6, pp. 1678-1686, Jun. 2008.

[15] S. Kidera, T. Sakamoto, and T. Sato, "Accurate UWB radar 3-D imaging algorithm for complex boundary without range points connections," IEEE Trans. Geosci. Remote Sens., vol. 48, no. 4, pp. 1993-2004, Apr. 2010.

[16] R. Bamler and M. Eineder, "The pyramids of Gizeh seen by TerraSAR$\mathrm{X}-\mathrm{A}$ prime example for unexpected scattering mechanisms in SAR," IEEE Geosci. Remote Sens. Lett., vol. 5, no. 3, pp. 468-470, Jul. 2008.

[17] J. M. F. Moura and Y. Jin, "Detection by time reversal: Single antenna," IEEE Trans. Signal Process., vol. 55, no. 1, pp. 187-201, Jan. 2007.

[18] G. Shi and A. Nehorai, "Cramer-Rao bound analysis on multiple scattering in multistatic point-scatterer estimation," IEEE Trans. Signal Process., vol. 55 , no. 6, pp. 2840-2850, Jun. 2007.

[19] E. A. Marengo, F. K. Gruber, and A. J. Devaney, "Generalized timereversal imaging considering multiple scattering effects," in Proc. IEEE Int. Symp. Antennas Propag., Aug. 2004, vol. 2, pp. 2087-2090.

[20] Federal Communications Commission (FCC), Office of Engineering and Technology (OET) Bulletin No. 65, Supplement C, p. 35, Aug. 1997.

[21] R. H. Stolt, "Migration by Fourier transform," Geophysics, vol. 43, no. 1, pp. 23-48, Feb. 1978.

[22] X. Xu, E. L. Miller, and C. M. Rappaport, "Minimum entropy regularization in frequency-wavenumber migration to localize subsurface objects," IEEE Trans. Geosci. Remote Sens., vol. 41, no. 8, pp. 1804-1812, Aug. 2003.

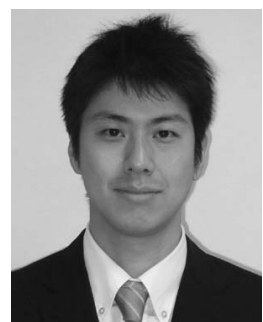

Shouhei Kidera (A'08) received the B.E. degree from the Faculty of Engineering, Kyoto University, Kyoto, Japan, in 2003 and the M.I. and Ph.D. degrees from the Graduate School of Informatics, Kyoto University, in 2005 and 2007, respectively.

$\mathrm{He}$ is currently an Assistant Professor with the Graduate School of Informatics and Engineering, The University of Electro-Communications, Chōfu, Japan. His current research interest is in advanced signal processing for the near-field radar and ultrawideband radar

Dr. Kidera is a member of the Institute of Electronics, Information, and Communication Engineers of Japan and the Institute of Electrical Engineers of Japan.

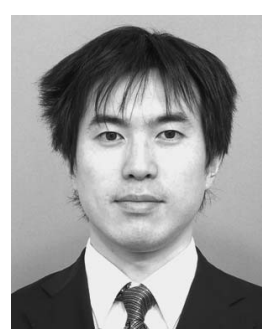

Takuya Sakamoto (M'04) was born in Nara, Japan, in 1977 . He received the B.E. degree from Kyoto University, Kyoto, Japan, in 2000 and the M.I. and $\mathrm{Ph}$.D. degrees from the Graduate School of Informatics, Kyoto University, in 2002 and 2005, respectively.

$\mathrm{He}$ is currently an Assistant Professor with the Department of Communications and Computer Engineering, Graduate School of Informatics, Kyoto University. His current research interest is in signal processing for ultrawideband pulse radars.

Dr. Sakamoto is a member of the Institute of Electronics, Information, and Communication Engineers of Japan and the Institute of Electrical Engineers of Japan.

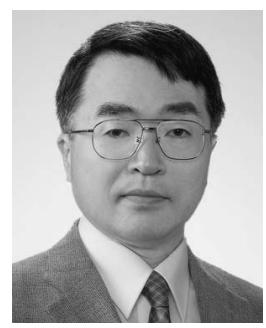

Toru Sato (M'92) received the B.E., M.E., and $\mathrm{Ph} . \mathrm{D}$. degrees in electrical engineering from Kyoto University, Kyoto, Japan, in 1976, 1978, and 1982, respectively.

Since 1983, he has been with Kyoto University, where he is currently a Professor in the Department of Communications and Computer Engineering, Graduate School of Informatics. His major research interests have been system design and signal processing aspects of atmospheric radars, radar remote sensing of the atmosphere, observations of precipitation using radar and satellite signals, radar observation of space debris, and imaging with ultrawideband pulse radars.

Dr. Sato was the recipient of the Tanakadate Prize in 1986. He is a fellow of the Institute of Electronics, Information, and Communication Engineers of Japan and a member of the Society of Geomagnetism and Earth, Planetary and Space Sciences, the Japan Society for Aeronautical and Space Sciences, and the American Meteorological Society. 